\title{
INFANTILE CEREBRAL GLIOSIS WITH GIANT NERVE CELLS
}

\author{
BY \\ L. CROME \\ From the Department of Neuropathology, the Fountain Hospital, Tooting, London
}

The usual form of congenital cerebral gliosis is characterized by induration and shrinkage of gyri and corresponding dilatation of the sulci. The shrinkage in each gyrus appears to be greater from side to side than from above downwards so that in severe cases the affected area is often walnut-like after the meninges are stripped. Histologically, there is neuronal loss and widespread fibrous and cellular gliosis. This is usually most marked in the marginal layer but may also be conspicuous in one of the deeper laminae, particularly the third. " Ulegyria ", a term signifying scarring of gyri, is generally applied to this condition. Its clinical features depend on the extent and localization of the lesion, and it is usually found in patients with varying degrees of mental retardation, microcephaly, motor disability, and epilepsy. In most of these there is no definite clinical or morphological evidence of a tendency to progress.

The object of this communication is to present three patients illustrating a somewhat different type of ulegyria. Of these two had come to necropsy and were examined pathologically. The third is still alive. In the two cases coming to necropsy wasting of the affected area was uniform. Although somewhat reduced in size, gyri tended to retain their usual outlines and contiguity, while the sulci were not unduly wide. The abnormality was not very obvious on inspection of the brain. Palpation was more useful, the affected area being very hard. Histologically there was marked loss of nerve cells and glial hyperplasia, most marked in the superficial cortical layers. Fibrous gliosis was diffuse in one case. In the second it was stratified and particularly conspicuous in the marginal and middle cortical layers. A striking morphological feature was the presence of large, irregularly shaped nerve cells with stout and tortuous processes. Many of the hyperplastic glial cells were also atypical. As in so many other cases of gross idiocy it was difficult to determine whether there was any clinical evidence of a progressive brain lesion. The head circumference was somewhat reduced in all three cases. They suffered from severe paralysis and severe intractable epilepsy.

\section{Case 1}

The patient was an illegitimate child of a woman aged 38 who had had four healthy older children by another father. The maternal grandparents were alive and well. The mother had tuberculosis of the knee, but pregnancy and confinement were normal. Nothing is known of the father and his family. The patient weighed $3.9 \mathrm{~kg}$. and appeared normal at birth.

Convulsions began at 2 months and were very frequent, the patient having up to $\mathbf{3 0}$ major and minor fits a day. These always started with twitching of the right eye, spreading to the right side and then to the rest of the body. She was admitted to hospital at $4 \frac{1}{2}$ months when increased tone and diminished power were noted in her right arm. The blood and C.S.F. findings were normal. An air encephalogram showed a well filled ventricular system, the left lateral ventricle being smaller than the right. An E.E.G. report read as follows: "It is difficult to distinguish purely epileptic activity from the normal at this age, but it is largely confined to the left side being of a character which would in later years be associated with idiopathic epilepsy. The possibility of an organic lesion can certainly not be ruled out."

Frequent fits continued in spite of heavy sedation and, in view of the possible presence of an epileptogenic lesion, it was decided to operate, the left frontal lobe being excised when the child was $7 \frac{1}{2}$ months old.

The excised material was embedded in paraffin. Some sections showed no abnormality, others presented slight focal proliferation of glial cells. A few of the nerve cells seemed irregular in shape and stained unevenly. Interpretation of these changes was difficult on account of widespread oedema, haemorrhages, and maceration produced by surgical handling, and the irregular shrinkage which is often seen in paraffin-embedded, freshly excised brain tissue. There was certainly no evidence of the striking abnormality found later at necropsy in the remaining part of the hemisphere.

The operation had only a transiently beneficial effect upon the fits. Their frequency was later somewhat reduced by "epanutin", but this was discontinued on account of increasing leucopenia. Some months after the operation there was flaccid paralysis of the right limbs, right facial palsy, and gross retardation of mental development. 
At the age of 2 she was admitted to the Fountain Hospital. At that time she had persistent twitching of the right facial muscles, the pupils were small and reacted sluggishly to light. The right arm and leg were spastic and the right plantar response extensor. She failed to respond to visual or auditory stimuli, made no articulate sounds and was unable to lift her head off the pillow. She died from bronchopneumonia at 2 years and 3 months, 20 months after the operation.

\section{Necropsy Report of Case 1}

Necropsy was performed 12 hours after death.

A healed scar was seen over the left frontal and parietal bones. The only other significant findings, apart from those in the nervous system, were in the lungs, which showed areas of lobular collapse, emphysema, and early bronchopneumonia. This was confirmed histologically. There was widespread alveolar collapse alternating with areas of inhalation pneumonia in which alveoli were filled with macrophages containing globules and granules of albuminous and fatty material. Many bronchioles were occluded by casts of desquamated epithelium and mucus and these casts extended, in some places, down into the alveoli. Other areas showed infiltration with inflammatory cells, chiefly polymorphs, and many alveoli were filled with clear homogeneous albuminous material.

Central Nervous System.-The brain without the surgically removed portion weighed $945 \mathrm{~g}$. The line of excision was at the level of the olfactory bulb upon the orbital surface reaching the inferior frontal gyrus $0.5 \mathrm{~cm}$. in front of the Sylvian fissure. It then curved upward and forward towards the frontal pole. Anteriorly, the cut surface was funnel-shaped with its centre drawn deeply inwards and communicating by a small opening with the tip of the anterior horn of the lateral ventricle. The whole agranular orbital cortex appeared to be spared and cortical loss on the convexity was confined to areas $9,10,46$, and 45 .

Considerable induration was present in the left hemisphere, the consistency of the right being normal. There was much discoloration of the cortex on the left side and demarcation between it and the subjacent white matter was absent. The anterior portion of the left centrum semiovale was somewhat friable and granular.

\section{Histological Examination in Case 1}

Coronal blocks of the frontal, parietal, temporal, and occipital lobes of both hemispheres, of the cerebellum, and of representative levels of the brain-stem and spinal cord were embedded in celloidin. Other blocks were embedded in paraffin and frozen material was also used as required. The customary general and neurological stains and impregnation methods were employed in the preparation of sections.

The most striking change was the presence of dense fibrous gliosis in the left cerebral hemisphere and its absence in the right. It was most marked anteriorly immediately at and behind the operation scar, diminishing steadily posteriorly, without, however, disappearing entirely. Fibrous gliosis affected, chiefly, the cortex and

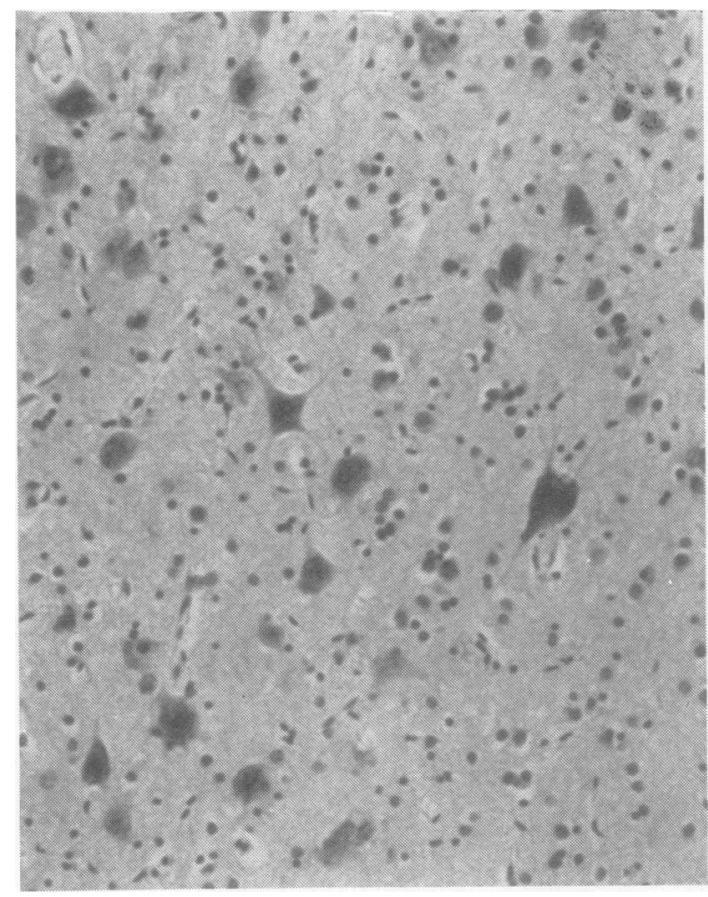

Fig. 1.-Numerous abnormally orientated giant cells. Some are nervous and others glial in origin. Cresyl violet $\times 200$.

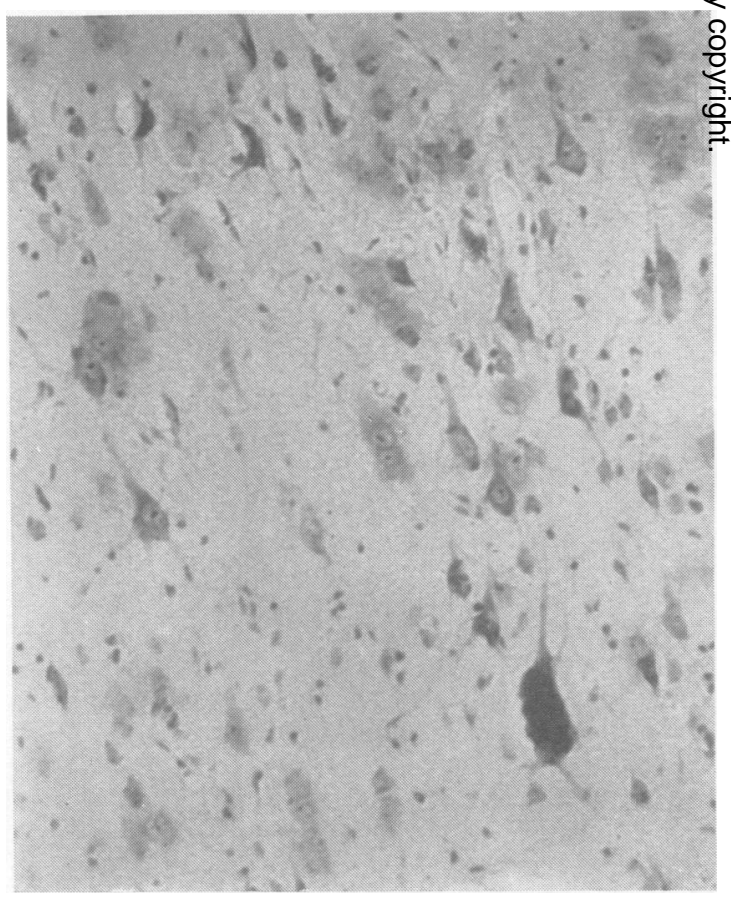

Fig. 2.-Many atypical glial cells and one giant nerve cell. Cresyl violet $\times 200$. 


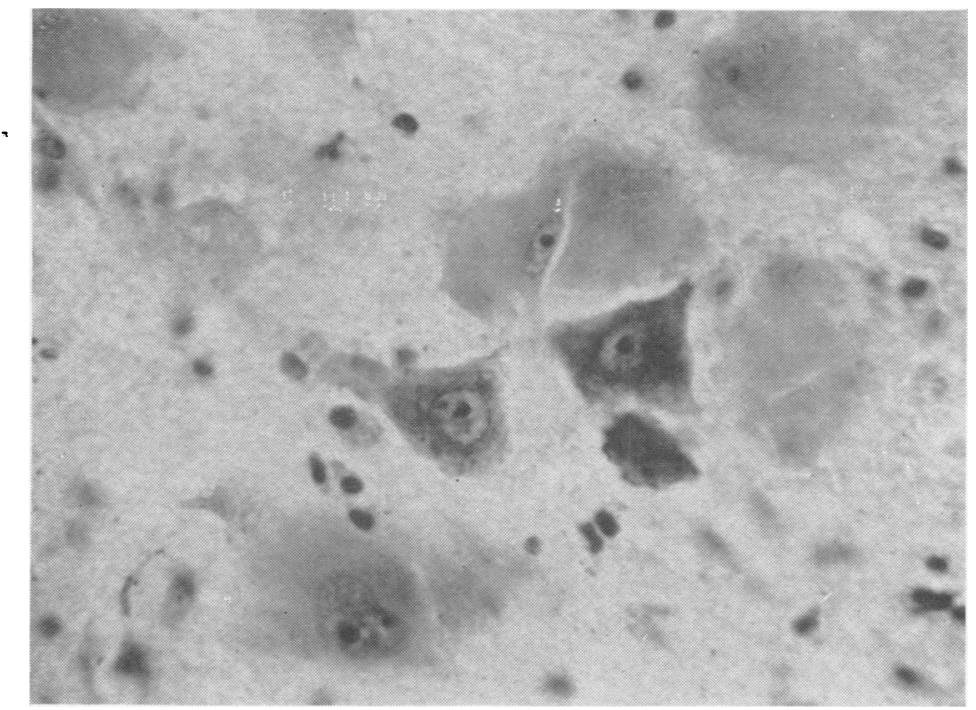

FIG. 3.-Atypical giant glial cells and some nerve cells. Cresyl violet $\times 550$.

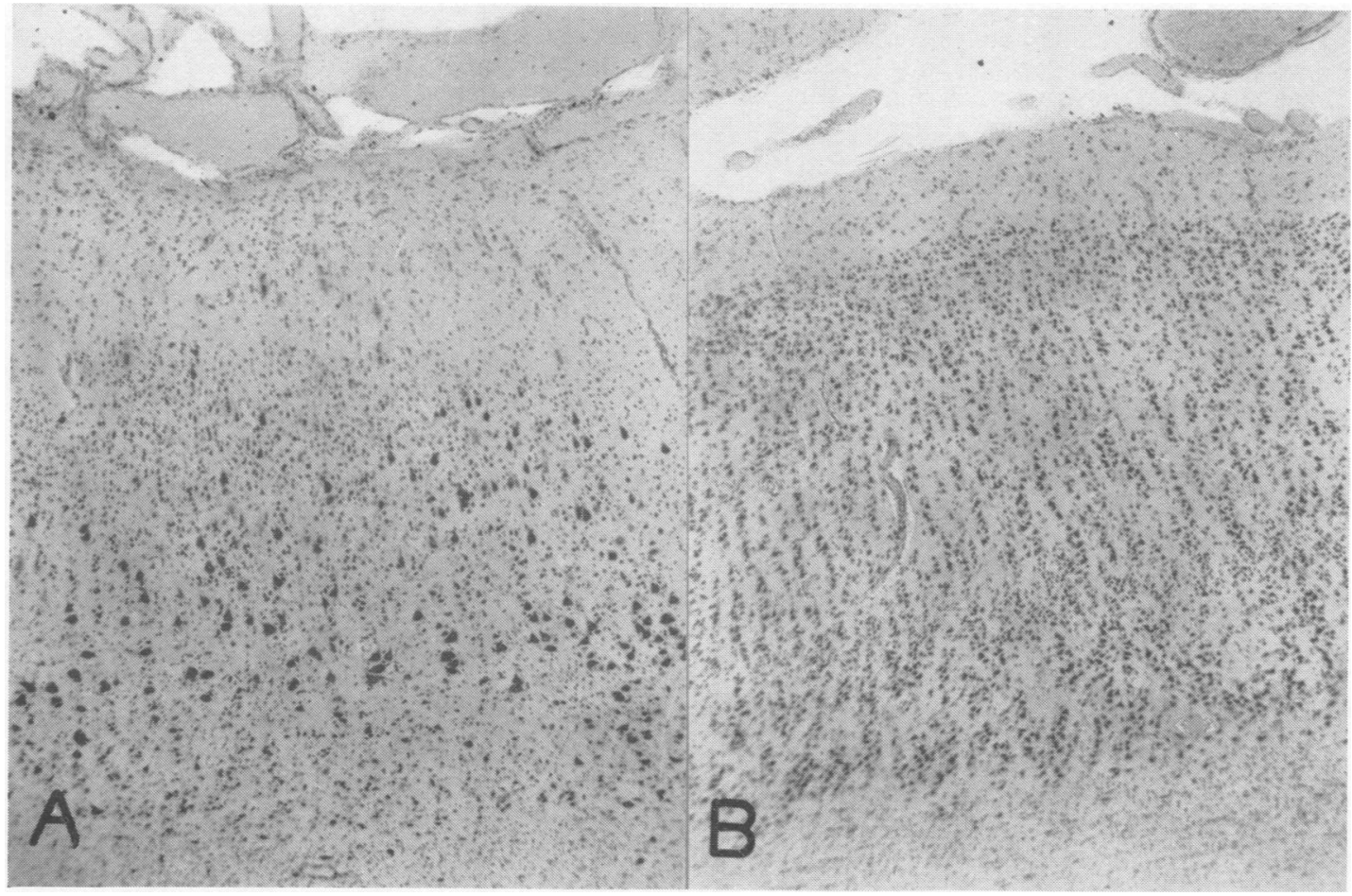

FIG. 4.-A, area 17 of the left hemisphere, and B, the same area of the right hemisphere. Cresyl violet $\times 50$ 
extended for a variable distance into the subjacent white matter. Cortical lamination was disrupted and totally unrecognizable in the worst areas. Nerve cells were reduced in number, those remaining showing many changes, the commonest being shrinking with pyknosis of both cytoplasm and nucleus or, by contrast, enlargement with distortion of the cell outlines and formation of giant cells (Fig. 1). Many of the latter were orientated at unusual angles. Bielschowsky preparations showed neurofibrils in a few cells. These fibrils were broken up into minute argyrophilic granules in some other cells. In most cells they were only discernible at the attachments of the axons and dendrites. But most of the cells were glial. Their structure varied greatly, in a manner defying brief description (Fig. 2 and 3). Many were protoplasmic with large irregular or oval bodies and vesicular or kidney-shaped nuclei. Some glial cells were as large or larger than the giant nerve cells and it was often impossible to distinguish between the two kinds of cells, although the former were, as a rule, paler with more distinct cell outlines and nuclear details. Another notable feature was the presence of many syncytial cells, "gliarasen", especially in the more superficial layers of the cortex, in the white matter, and in the basal ganglia. Minute granules of sudanophil material were seen in some of the glial and nerve cells while larger particles of neutral fat were present around occasional blood vessels and in the endothelial cells of some capillaries. A few round or oval granules of amorphous calcified material were also present near blood vessels in some areas.

Changes in the more posterior parts of the left hemisphere were similar but less marked. Here the cortical lamination was better preserved but the abnormality was recognized by the presence of single or focal collections of abnormal cells and fibrous gliosis. There was, in addition, a reduction in the density of the nerve cells, particularly in the superficial layers.

The hippocampus showed some marginal fibrous gliosis, which was also present in all other parts of the brain, and fibrous gliosis of the white matter. There was no detectable loss of nerve cells in the Ammon's horn.

The myelin of the white matter was well preserved posteriorly, but there was considerable demyelination in the anterior part of the centrum semiovale with fibrous gliosis and proliferation of large glial cells, particularly of the "gliarasen" type. These were also conspicuous in the left basal ganglia which were severely affected and showed almost total disappearance of nerve cells in all their constituent nuclei.

Changes in the right hemisphere bore a totally different character. Cortical lamination was preserved everywhere, albeit rather indistinctly in certain areas. The density of the nerve cells was reduced uniformly in layers II and III (Fig. 4), and patchily in the infragranular ones. There was a moderate increase in the number of astrocytes and microglia in the molecular layer. Many nerve cells showed, like the surviving ones in the opposite hemisphere, central chromatolysis and homogenizing change. Marginal fibrous gliosis was also present. Giant nerve cells were absent. The white matter showed some fibrous gliosis without demyelination in all areas except the more anterior portion of the centrum semiovale, where

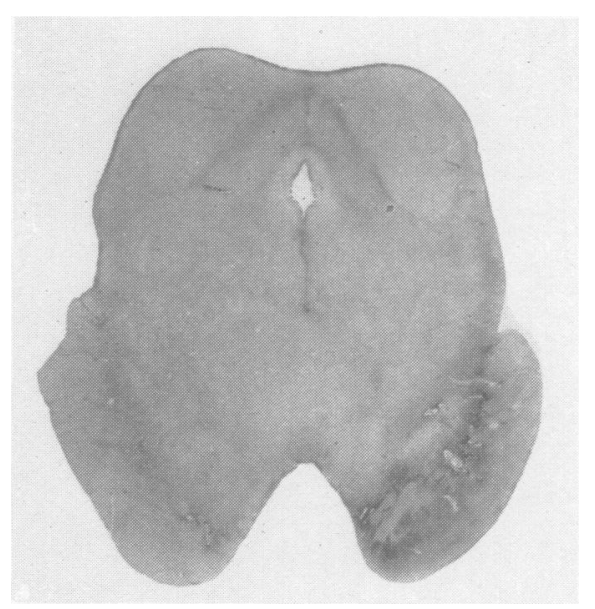

FIG. 5.-Fibrous gliosis in left peduncle of the mid-brain. Holzer $\times 3$.

it was accompanied by some demyelination. The nuclei of the basal ganglia were also well preserved, and fibrous gliosis was present only in the external capsule and the area between the amygdaloid and striate nuclei. number of Alzheimer type I cells were present in the globus pallidus.

Dense fibrous gliosis was present in the white matteo of the cerebellum. There was also a slight reduction io the number of Purkinje cells and in the density of the granular layer.

The left cortico-spinal tract showed lack of myelinatio throughout its entire length in the midbrain, ponse $\vec{c}$ medulla, and opposite lateral column of the spinal cord There was also diffuse fibrous gliosis affecting the whole of the brain-stem and this was most marked in areas corresponding to the left pyramidal tract (Fig. 5), as well as around blood vessels and the gray matter of the spinal cord.

\section{Case 2}

This girl was a first child, born after a normal pregnancy and confinement. The family history was negative. She was diplegic and started having fits soon after birth. These were described as beginning in the right hand and spreading to the whole body. She had left facial palsy and generalized hypertonia with exaggerated reflexes of all limbs. The head circumference was $39.9 \mathrm{~cm}$. Tapping for subdural haematoma proved fruitless; the C.S.F. was normal and the blood calcium level not raised.

A year later she was admitted twice to hospital within two months for pneumonia. At that time it was plain that she was mentally retarded.

At 22 months she was admitted to the Fountain Hospital. She was then an inert, fat child with a head circumference of $43.2 \mathrm{~cm}$. and mid-thoracic scoliosis. She had an inconstant divergent squint, moderate general of spasticity with increased tendon jerks, absent abdominal reflexes, extensor plantar responses, and left ankle clonus. Her vision and hearing were doubtful. She had many fits every day, not relieved by drugs. She remained 
unaware of her surroundings, lying flat on her back and making few movements. She was thought to be of idiot mental level.

She developed respiratory difficulties soon after admission. Fits continued unabated and her feeding was very difficult. She had frequent cyanotic attacks and died from bronchopneumonia at 2 years.

\section{Post-mortem Examination in Case 2}

The body was that of an underdeveloped girl (height $85 \mathrm{~cm}$., weight $9.0 \mathrm{~kg}$.), showing generalized wasting and marked scoliosis. The only other significant somatic finding was patchy consolidation of the lungs presenting bronchopneumonic changes on microscopical examination.

Central Nervous System.-The brain was small (560 g.) with the meninges at the base somewhat opaque. The cerebellum with the brain-stem were relatively large, weighing $100 \mathrm{~g}$. The pattern of convolutions was normal, the left temporal lobe being, however, slightly smaller than the right (Fig. 6 and 7). Marked induration was

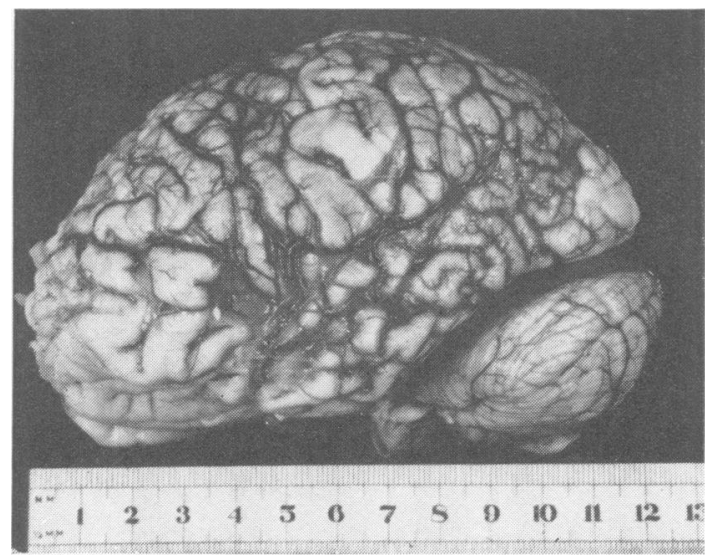

FIG. 6

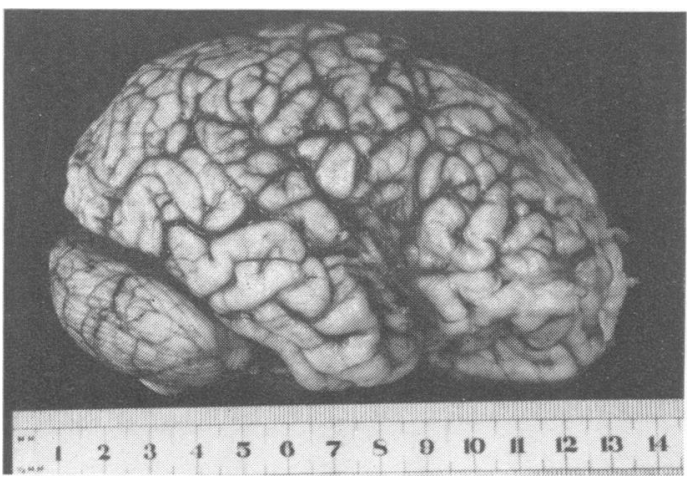

FIG. 7

Fig. 6.-Left hemisphere: moderate atrophy of temporal and lower half of parietal and occipital lobes.

FIG. 7.--Right hemisphere.

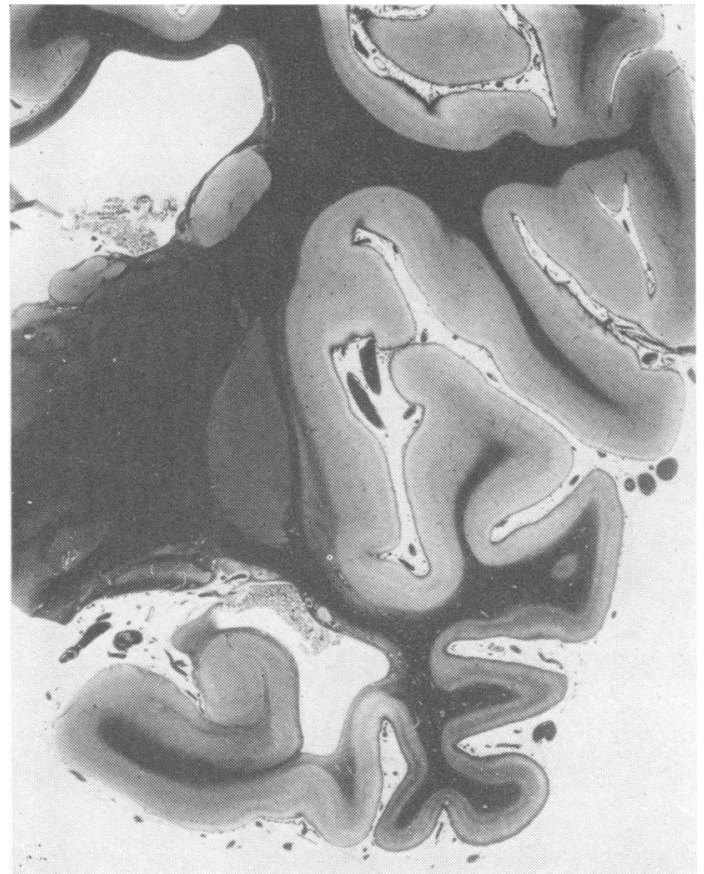

Fig. 8.-Temporal lobe: fibrous gliosis of cortex most marked in the molecular and middle cortical layers. Heidenhain $\times 4$.

present in the left temporal lobe and posterior to it, extending all the way to the occipital pole. The right hemisphere was normal in consistency. The ventricles showed moderate dilatation, most marked in the inferior horn of the left lateral ventricle. The corpus callosum was thin. The basal ganglia, cerebellum, and brain-stem presented no naked-eye abnormality.

The histological methods employed were as in Case 1 above. The main changes consisted of fibrous gliosis and neuronal loss in the left middle and inferior temporal gyri, and fusiform gyrus, but not the hippocampus (Fig. 8). Posteriorly, similar changes were present in the lower half of the lateral surface of the parietal and occipital lobes. This abnormality was uniform over the whole extent of the affected area. Fibrous gliosis was most conspicuous in the molecular layer and, again, in a position corresponding with the second, third, and fourth layers. The subjoining white matter was also affected by dense gliosis, showing in addition demyelination and glial cellular hyperplasia.

The cytoarchitectonic pattern was also fairly uniform over the entire diseased area, presenting marked cellular loss in the deeper portion of the second and in the third and fourth layers (Fig. 9). The superficial parts of the external granular and of the fifth and sixth layers were better preserved. This pattern corresponded with the distribution of fibrous gliosis. The molecular layer of the gliosed cortex showed an increase in the number of glial cells-mostly fibrillary astocytes (Fig. 10). Some 


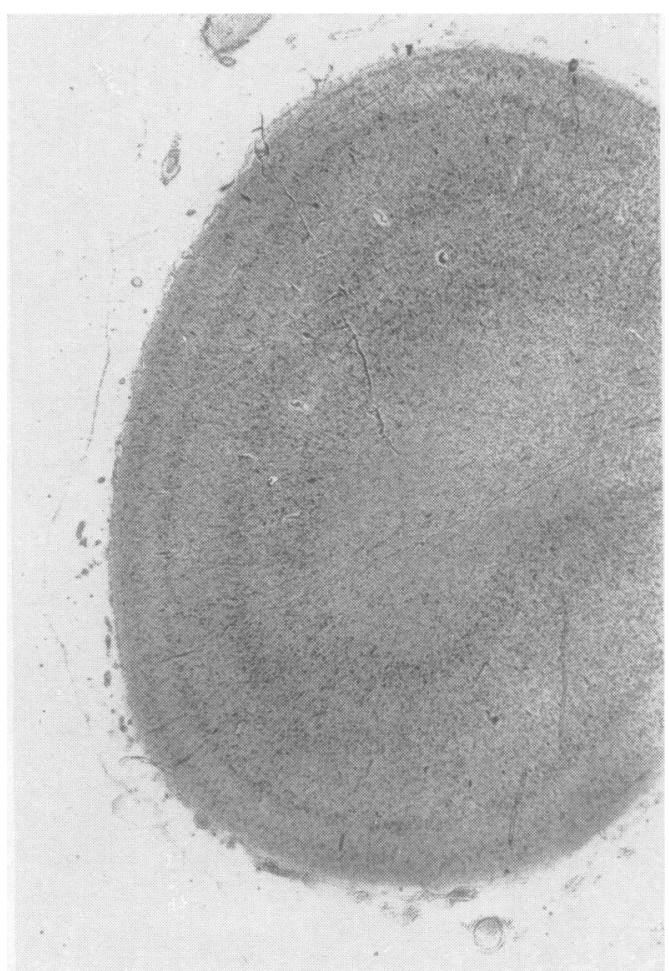

FIG. 9.-Loss of nerve cells in cortex of temporal lobe. Cresyl violet $\times 17$.

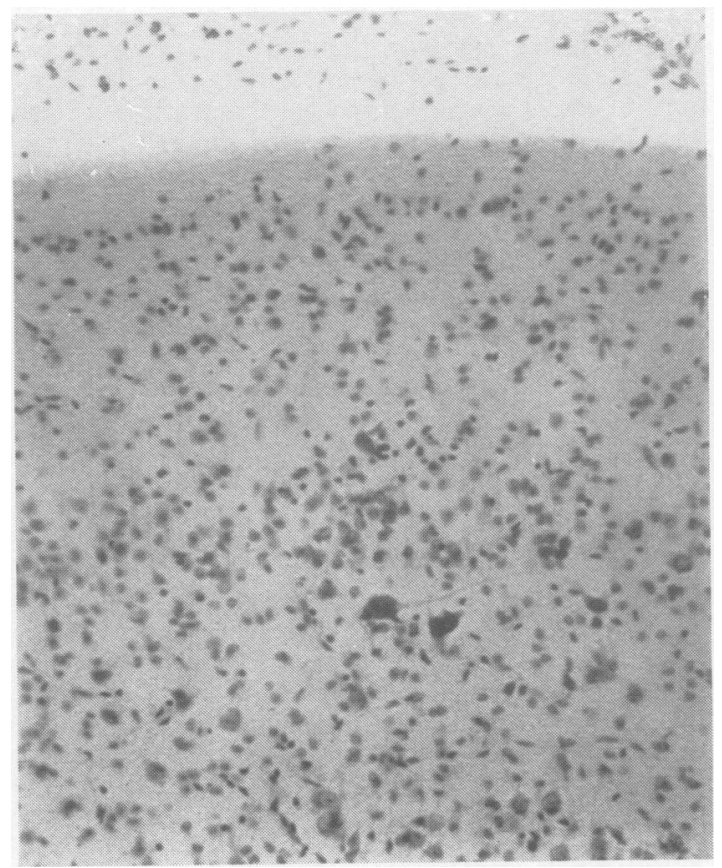

FIG. 10.-Glial hyperplasia in molecular layer: two abnormally orientated giant nerve cells in layer III. Cresyl violet $\times 160$.

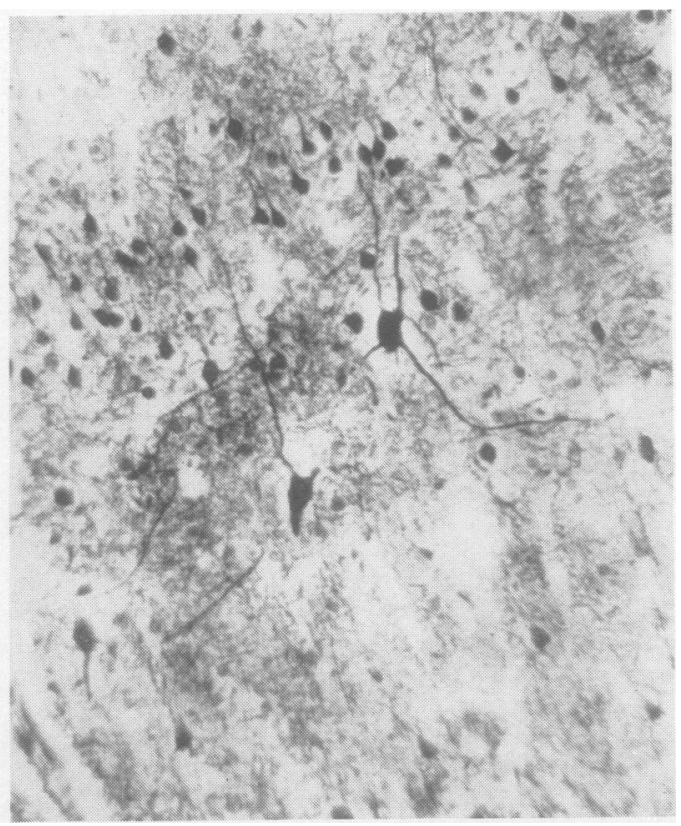

Fig. 11.-Two giant nerve cells. Bielschowsky $\times 160$.
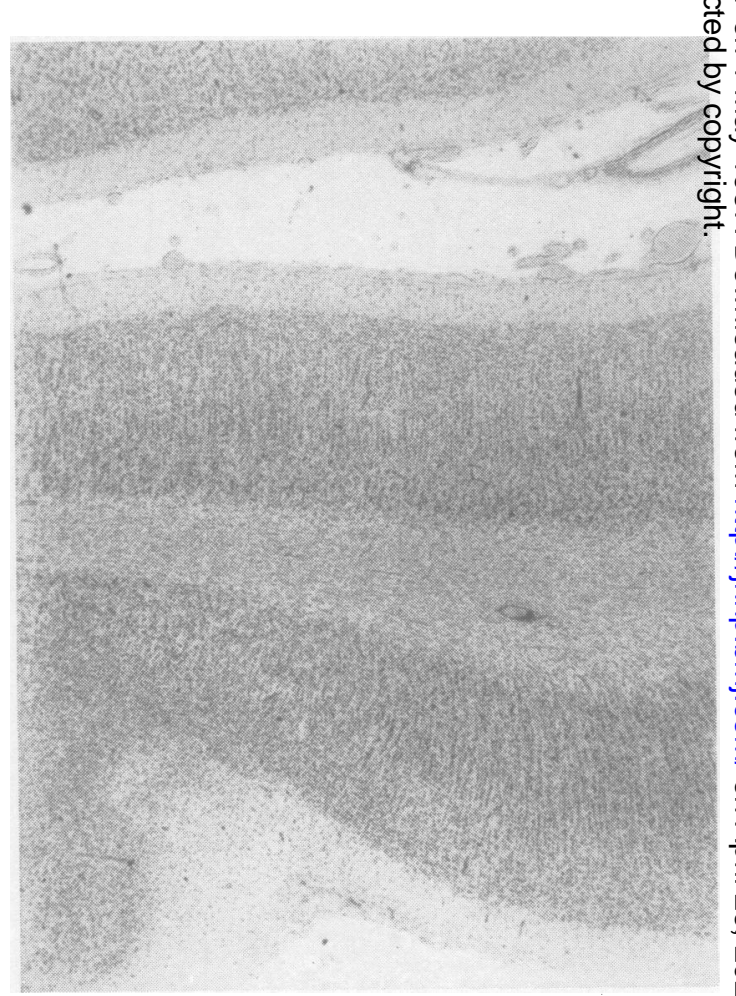

FIG. 12.-Diffuse loss of nerve cells and blurring of cytoarchitectonic pattern in one of the " unaffected" areas. Cresyl violet $\times 17$. 
of the surviving nerve cells in layers 3 and 4 were excessively large and orientated at unusual angles, and had thick and tortuous processes (Fig. 11). The neurofibrillary structures impregnated badly with silver, and it proved impossible to decide which of the cellular processes were axons or dendrites. Many of the surviving nerve cells stained unevenly and "ghost" forms were also present. A few microglial cellular clusters were scattered throughout the gray matter of the central nervous system. A moderate amount of neutral fat could be seen in the affected cortex and white matter. Some of it was intracellular, included in the cytoplasm of compound granular corpuscles and endothelial capillary cells, but free fatty particles were also present.

The other remaining areas in both hemispheres showed considerably milder changes: some glial hyperplasia in the molecular layer, blurring of the cytoarchitectonic pattern and a fairly uniform diffuse loss of nerve cells in the superficial cortical layers (Fig 12). The white matter in these areas showed slight fibrous gliosis without perceptible myelin loss. There was a slight reduction of nerve cells in the nucleus ventralis posterior and nucleus medialis dorsalis of the left thalamus. Other thalamic nuclei, on both sides, presented no abnormality. Changes in the cerebellum consisted of slight glial hyperplasia in the molecular layer and the presence of Bergmann glia. There was no atrophy in the Purkinje and granular layers. Periventricular fibrous gliosis was present in the ventricles. Marginal fibrous gliosis affected the brainstem and spinal cord. There was no demyelination of the cortico-spinal tracts.

\section{Case 3}

This patient was a sister of Case 2, born three years later after a normal pregnancy and confinement. This child had minor epilepsy from the age of 1 month. The fits were at first tonic and accompanied by a leftward deviation of the head. At 6 months they became akinetic with no apparent loss of consciousness, a deviation of the eyes to the right or left, and rapid, panting breathing. The fundi were pale but the optic discs normal. Muscle tone was slightly raised in the right leg. An air ventriculogram showed some widening of the sulci in the right frontal region, suggesting cerebral atrophy. Electroencephalographic findings were compatible with diffuse cortical atrophy. The C.S.F. and other laboratory tests were normal.

At the time of writing the child is a year old. In spite of medication she is having four or five attacks of epilepsy every day. The skull circumference is $43.4 \mathrm{~cm}$. and weight $11.5 \mathrm{~kg}$. She has an asymmetrical face with the fontanelle open $2.4 \mathrm{~cm}$. The optic discs are normal in colour, there is generalized retinal hypoplasia, but no choroidoretinitis or macular change. Muscular tone is raised, tendon jerks exaggerated, and plantar responses extensor. Her mental development is greatly retarded.

\section{Discussion}

The chief clinical features in the three patients were intractable epilepsy, paralysis, and idiocy. The morphological findings in the two cases coming to necropsy were characterized by diffuse atrophy and gliosis affecting large but restricted areas of the cerebral cortex and the subjoining white matter. Giant nerve cells were present in both cases, being more numerous in the first. A substantial amount of neutral fat, disappearing nerve cells, and glial hyperplasia were suggestive of continued structural degeneration at the time of death.

The details of the histological picture and, particularly, the giant nerve cells, were reminiscent of tuberous sclerosis. It seems likely, indeed, that some of the previously published records of so-called unilateral and localized tuberous sclerosis (Jakob, 1914) referred to similar cases. It is, however, improbable that the condition is tuberous sclerosis. The changes in these cases are diffuse rather than nodular, while candle guttering as well as all the other characteristic somatic manifestations of tuberous sclerosis are absent.

The changes in the second case resemble those described by Schob (1930) as " progressive sclerosing hemiatrophy ", other instances of which were later reported by Töppich (1935) and Josephy (1945). Schob wrote that in this condition there is fairly uniform progressive parenchymal loss seen chiefly in one of the cortical laminae, especially the third, and corresponding fibrous gliosis. Clinically, he defined the condition as one of increasing mental deterioration, epilepsy, and paralysis. Although designated as "hemiatrophy" the extent of the changes may be more limited, affecting only one or several lobes. Case 2 corresponds quite well with this description but not Case 1, where localization of neuronal loss and of gliosis was not confined or specially conspicuous in any one of the cortical laminae.

The occurrence of giant nerve cells in different sclerotic conditions has also been mentioned by Schob. As stated already, they are a special and familiar feature of tuberous sclerosis. They are, however, non-specific and known to be associated with other conditions, such as pachygyria (Crome, 1956). Their origin and significance are obscure.

All the cases were probably congenital, but apart from this and their morphological likeness there is no evidence that they were due to the same cause. It is likely that the sclerotic process in the first case was accentuated, if not initiated, by the lobectomy. It is difficult to explain in any other way the absence of similar changes in the part of the frontal lobe removed at operation and in the second hemisphere, and, also, the receding severity of the lesion from the line of excision. The second and third cases were siblings and thus, presumably, identical in aetiology. 
It is possible that some of the changes were themselves the result of frequent uncontrollable fits but here one cannot be certain of what is cause and effect. It may be significant that lesions commonly attributed to epilepsy, viz., loss of nerve cells in the Sommer sector of the hippocampus and in the Purkinje layer of the cerebellum, as well as the degeneration of the globus pallidus (Scholz 1933, 1936), were either entirely absent or not particularly marked in the two fatal cases.

\section{Summary}

Some gliotic processes of the brain are characterized by diffuse, non-nodular atrophy of the cortex and subjoining white matter. The lesions are diffuse and extensive but do not affect the whole of the brain, the remaining parts being free from similar change. Histologically, there is cellular glial hyperplasia, fibrous gliosis, and loss of nerve cells. These may be particularly marked in the molecular and in one of the deeper laminae, especially the third. Another striking feature is the presence of irregularly orientated giant nerve cells having stout and tortuous processes. The clinical manifestations of the condition include cerebral palsy, severe epilepsy, and a very low mental and physical level of attainment. The condition is probably congenital but its aetiology and pathogenesis are otherwise obscure. This communication is based on two patients who had come to necropsy and a third still alive.

I wish to record my indebtedness to my lay and medical colleagues at the Fountain and other hospitals for their cooperation and access to case notes.

\section{REFERENCES}

Crome, L. (1956). J. Path. Bact., 71, 335.

Jakob, A. (1914). Z. ges. Neurol. Psychiat. (Orig.), 23, 1.

Josephy, H. (1945). J. Neuropath., 4, 250

Schob, F. (1930). Handbuch der Geisteskrankheiten, ed. Bumke, O. Band XI Spezieller teil VII. Die Anatomie der Psychosen, ed. Spielmeyer, M., p. 929. Springer, Berlin.

Scholz, W. (1933). Z. ges. Neurol. Psychiat., 145, 471.

- (1936). Allg. Z. Psychiat., 104, 89.

Töppich, G. (1935). Arch, Psychiat. Nervenkr., 103, 335.

\section{THE FEBRUARY (1957) ISSUE}

The February (1957) issue contains the following papers:-

Abnormal Immunological Reactions in Disseminated Sclerosis. Honor V. Smith, M. L. E. Espir, C. W. M. Whitty, and W. Ritchie Russell.

Loss of Recent Memory after Bilateral Hippocampal Lesions. W. B. Scoville and Brenda Milner.

The Recurrence of Intracranial Meningiomas after Surgical Treatment. Donald Simpson.

A Variety of Paramyotonia Congenita. E. B. French and R. Kilpatrick.

A Simplified Neurosurgical Technique for Approaching and Damaging the Region of the Globus Pallidus in Parkinson's Disease. S. Obrador.

An Unusual Form of Widespread Vascular Disease of the Brain in a Youth. C. S. Treip and R. J. Porter.

Some Relationships between Peripheral Vasomotor and E.E.G. Changes. Brian Ackner and G. Pampiglione.

Conduct Disorders in Epileptic Children. F. Grunberg and D. A. Pond.

Proceedings of the Society of British Neurological Surgeons.

Book Reviews.

A number of copies are still available and may be obtained from the Publishing Manager, British Medical Association, Tavistock Square, W.C.1, price 12s. $6 d$. 